\title{
Intuitiveness, quality and utility of intraoperative fluorescence videoangiography: Australian Neurosurgical Experience
}

\author{
VINI G. KHURANA ${ }^{1,2}$, KEVIN SEOW ${ }^{1} \&$ DAVID DUKE $^{3}$ \\ ${ }^{1}$ Departments of Neurosurgery and ${ }^{3}$ Anaesthetics, The Canberra Hospital, ${ }^{2}$ Australian National University Medical School, \\ Canberra, ACT, Australia
}

\begin{abstract}
Introduction: The authors have undertaken a study of their intraoperative experience with indocyanine green fluorescence videoangiography (ICGFV). In particular, the intuitiveness, image quality and clinical utility of this technology have been assessed.

Methods: The records of forty-six consecutive craniotomies utilising ICGFV have been retrospectively reviewed: There were 27 aneurysms, 2 extracranial-intracranial (EC-IC) bypasses, 5 arteriovenous malformations (AVM), 1 dural arteriovenous fistula (DAVF), 3 cavernomas, 5 meningiomas, and 3 gliomas. ICGFV was used in 5 awake-craniotomy patients. ICGFV was performed using a Leica OH4 surgical microscope with integrated near-infrared camera and ICG-PULSION.

Results: All attempts of intraoperative ICGFV were intuitive. Image quality and resolution were excellent. Arterial and venous phases were comparable to digital subtraction angiography (DSA) but field of view was relatively limited. In 12 operations $(26 \%)$ the surgeon was substantially benefited from ICGFV findings. In 22 operations (48\%), ICGFV was useful but did not influence surgical management. ICGFV was of no benefit in 11 operations (24\%) and was misleading in 1 ( $2 \%)$. In this series, ICGFV was of benefit to 1 of $11(9 \%)$ patients with an intracranial neoplasm or cavernoma.

Conclusions: ICGFV is safe, intuitive and provides neurosurgeons with high quality, valuable, real-time imaging of cerebrovascular anatomy. It can assist in intraoperative surgical management and/or stroke prevention particularly during aneurysm clipping, EC-IC bypass and AVM/DAVF surgery.
\end{abstract}

Key words: Arteriovenous malformation, Dural arteriovenous fistula, Extracranial-intracranial bypass, Fluorescence videoangiography, Indocyanine green, Intracranial aneurysm.

\begin{abstract}
Abbreviations: AVM, arteriovenous malformation; CTA, computerised tomographic angiography; DAVF, dural arteriovenous fistula; DSA, digital subtraction angiography; EC-IC, extracranial-intracranial; ICGFV, indocyanine green fluorescence videoangiography; ITTF, intraoperative transit time flowmetry; NIR, near-infrared.
\end{abstract}

\section{Introduction}

Indocyanine green fluorescence videoangiography (ICGFV) is an emerging intra-operative neurovascular imaging modality. This technique was first approved for cardiac circulatory assessment, liver function diagnostic use and ophthalmic angiography. In recent years, it has been adopted into neurosurgery with large centres in Germany, USA and Japan reporting positive experience with this technology. ${ }^{1-8}$ However, its use in Australia, New Zealand and UK neurosurgical centres has not been widely documented.

The use of intra-operative ICGFV has been reported to provide surgeons with timely and important information leading to changes in intraoperative surgical management. In one series, aneur- ysm clip repositioning based on ICGFV findings occurred in $9 \%$ of cases. ${ }^{4}$ In addition, the results of ICGFV have been shown to correspond to intra- and post-operative digital subtraction angiography (DSA) in $90 \%$ of cases. ${ }^{4}$

ICGFV uses an iodine-based compound that fluoresces at near-infrared (NIR) exposure (wavelength 780-950 $\mathrm{nm}$ ). The emitted fluorescence is detected, filtered and relayed into a processing unit providing real-time high-quality videoangiography. In its current form as an integrated NIR cameramounted on the Leica $\mathrm{OH} 4$ or Zeiss Pentero microscope, ICGFV is easy to set up, simple to use and requires minimal additional resources. ${ }^{2-4}$

The purpose of this study is to report the seminal Australian experience with the use of intra-operative ICGFV from the perspectives of intuitiveness of the 
technology, image quality in comparison to the gold standard of DSA along with its clinical benefit. We also attempted to determine whether there was any benefit of using this technology in various selected non-cerebrovascular neurosurgical settings. To our knowledge, this study reports the broadest utility of this technology in neurosurgery in a single study. Furthermore, we report the first use of ICGFV in awake craniotomies $(n=5)$.

\section{Methods}

This is a retrospective study involving the review of office and operative records of all craniotomies carried out between August 2007 and August 2009 by the corresponding author (VGK) in which ICGFV technology was used. For the purposes of generating a written report to the Australian Government's Therapeutic Goods Administration (TGA) that approved the seminal neurosurgical use of this technology in Australia, relevant experience data from each use of ICGVA had been prospectively added to a computer database by two of the authors (VGK, KS).

\section{Patient population}

The records of 44 consecutive patients undergoing a total of 46 craniotomies utilising ICGFV were reviewed. The craniotomies were: $n=27$ for aneurysm clipping (including one 2-stage operation for bilateral aneurysms), $\mathrm{n}=2$ for EC-IC bypass, $\mathrm{n}=3$ for cavernous malformation removal, $n=5$ for AVM excision (including one re-operation), $n=1$ for DAVF disconnection, $\mathrm{n}=5$ for meningioma resection, and $n=3$ for glioma resection. Five of the 44 patients were administered ICG whilst undergoing awake craniotomy. Written informed consent had been received from each of the patients undergoing elective ICGFV usage. A comprehensive consent form pertaining to this technology had been developed according to the advice received from the Australian Therapeutic Goods Administration (under Special Access Scheme category B No. 2007/ 03081; approved June 15, 2007) and The Canberra Hospital Medical Records Department.

In the emergency setting, provided that the patient had no history of iodine allergy, ICGFV was used at the discretion of the neurosurgical team with foremost consideration of the patient's best interest and whenever possible with the additional approval of the patient's next-of-kin. ICGFV was used for all cerebrovascular cases (including cavernous malformations) operated by the corresponding author following acquisition of the technology and its approval. ICGFV was used in a limited number of non-cerebrovascular neurosurgical cases on the basis of complexity and anticipated proximity to large vascular structures such as dural venous sinuses or circle of Willis arteries on pre-operative planning.
The use of ICGFV in awake patients allowed realtime observation of any adverse symptoms or change in sensorium of the patient during ICG administration.

\section{Equipment}

ICGFV was carried out using a Leica M525 OH4 surgical microscope with integrated near-infrared camera FL 800 (Leica Microsystems, Switzerland).

\section{Indocyanine green (ICG)}

ICG is a water-soluble, iodide-containing tricarbocyanine dye with a peak spectral absorption at 800$810 \mathrm{~nm}$ in blood or plasma. ICG was acquired from PULSION Pacific (Alexandria NSW, Australia; subdivision of PULSION Medical Systems, Munich). ICG-PULSION was supplied in $25 \mathrm{mg}$ or $50 \mathrm{mg}$ vials of powder ( 5 vials per box). The authors had utilised $50 \mathrm{mg}$ vials diluted with $10 \mathrm{~mL}$ of sterile injectable water, thereby constituting a 5 $\mathrm{mg} / \mathrm{mL}$ preparation. The dose of ICG-PULSION administered had been optimised for intraoperative use by the anaesthetist (DD) and a bolus dose of 0.15 $\mathrm{mg} / \mathrm{kg}$ (with a maximum cumulative total of $5 \mathrm{mg} / \mathrm{kg}$ ) was administered intravenously through a peripheral or central line followed by normal saline flush. Typically, a total of 2 to 5 injections of ICGFV (all derived from the single $50 \mathrm{mg}$ ICG vial) were used in cerebrovascular cases. The cost of ICG per $50 \mathrm{mg}$ vial during this study was approximately AUD $\$ 150$.

\section{Assessment of safety and reliability of ICG/ICGFV}

ICG is regarded as a clinically safe dye based on its use for decades in the fields of cardiology, hepatology and ophthalmology. The principle contraindication to its use is known iodine hypersensitivity. The occurrence of serious hypersensitivity reactions to ICG in patients without a history of allergy to iodine is extremely rare. None of the 44 patients undergoing ICGFV had a history of iodine allergy. Safety of ICGFV was assessed by the authors via documentation of any adverse (hypersensitivity) reaction to ICG, including any reported adverse symptoms during awake craniotomy ICG administration, any substantial impact on operative time, or any operational or mechanical problems in the Leica OH4 operating microscope during videoangiography. Reliability of ICGFV had been assessed by comparing intraoperative ICGFV findings to routine postoperative digital subtraction angiography (DSA) or computerised tomographic angiography (CTA) and the patient's neurological outcome.

\section{Results}

There were 30 females and 14 males. The average age was 48.6 years (range 17-77 years). Thirty-two 
procedures were elective and 14 were emergencies (Table I). All ICGFV attempts were successful. There were no technical or mechanical impediments during ICGFV or failed intended visualisations of the fluorescence in the surgical field. There were no complications, hypersensitivity or other side effects observed from the use of ICGFV. The use of ICGFV added less than 10 minutes to the operation time. The 5 awake craniotomy patients did not report any discomfort or dysaesthesia, and no altered sensorium was observed in these patients, during or following intravenous (IV) injection of ICG. Except for 1 patient with an AVM requiring the use of a second
$50 \mathrm{mg}$ vial, each patient typically used less than 1 vial of ICG preparation.

\section{Intuitiveness and simplicity}

The authors found the Leica $\mathrm{OH} 4$ microscope with integrated NIR-camera easy to use. With a flick of a switch, the microscope readily changed to the fluorescence videoangiography mode. The primary neurosurgeon was able to visualise and coordinate the real image in one eyepiece and the fluorescence image in the other eyepiece (Fig. 1). All other viewing eyepieces on the microscope were confined

TABLE I. Study population and usefulness of ICGFV

\begin{tabular}{|c|c|c|c|c|}
\hline Craniotomy & Age, Sex & Surgery & Type & Benefit ${ }^{\star}$ \\
\hline 1 & $33 \mathrm{~F}$ & ICA-OA aneurysm & Elective & 2 \\
\hline 2 & $17 \mathrm{~F}$ & P1 pseudoaneurysm & Emergency & 3 \\
\hline 3 & $43 \mathrm{~F}$ & Distal ICA fusiform aneurysm & Elective & 3 \\
\hline 4 & $40 \mathrm{M}$ & Falcine meningioma & Elective & 1 \\
\hline 5 & $45 \mathrm{M}$ & Distal ICA aneurysm & Elective & 2 \\
\hline 6 & $39 \mathrm{~F}$ & EC-IC bypass with RA graft & Elective & 3 \\
\hline 7 & $27 \mathrm{M}$ & Insular astrocytoma - awake & Elective & 1 \\
\hline 8 & $44 \mathrm{~F}$ & Anterior choroidal artery aneurysm & Emergency & 2 \\
\hline 9 & $63 \mathrm{M}$ & Broca cavernoma - awake & Elective & 1 \\
\hline 10 & $65 \mathrm{~F}$ & A-comm aneurysm & Emergency & 3 \\
\hline 11 & $45 \mathrm{~F}$ & Midline clival meningioma & Elective & 1 \\
\hline 12 & $22 \mathrm{~F}$ & SM Grade 3 insular AVM; ruptured & Elective & 4 \\
\hline 13 & $22 \mathrm{~F}$ & SM Grade 3 insular AVM; residual & Elective & 1 \\
\hline 14 & $63 \mathrm{~F}$ & MCA aneurysm & Elective & 2 \\
\hline 15 & $56 \mathrm{~F}$ & A-comm aneurysm & Emergency & 2 \\
\hline 16 & $24 \mathrm{~F}$ & Internal capsule cavernoma - awake & Elective & 1 \\
\hline 17 & $64 \mathrm{~F}$ & Parietal cortical DAVF & Emergency & 3 \\
\hline 18 & $30 \mathrm{~F}$ & P-comm aneurysm & Emergency & 2 \\
\hline 19 & $72 \mathrm{~F}$ & Olfactory groove meningioma & Elective & 1 \\
\hline 20 & $37 \mathrm{~F}$ & Frontal GBM & Elective & 1 \\
\hline 21 & $40 \mathrm{M}$ & MCA aneurysm & Emergency & 1 \\
\hline 22 & $43 \mathrm{~F}$ & Falcine meningioma & Elective & 1 \\
\hline 23 & $64 \mathrm{~F}$ & A2-A3 aneurysm & Elective & 3 \\
\hline 24 & $77 \mathrm{M}$ & EC-IC bypass with RA graft & Elective & 2 \\
\hline 25 & $58 \mathrm{~F}$ & A-comm aneurysm & Emergency & 2 \\
\hline 26 & $68 \mathrm{~F}$ & MCA aneurysm & Elective & 3 \\
\hline 27 & $48 \mathrm{M}$ & PICA aneurysm & Emergency & 2 \\
\hline 28 & $50 \mathrm{~F}$ & A-comm aneurysm & Emergency & 2 \\
\hline 29 & $50 \mathrm{~F}$ & Distal ICA aneurysm & Emergency & 2 \\
\hline 30 & $55 \mathrm{M}$ & Proximal ICA dissecting aneurysm & Emergency & 2 \\
\hline 31 & $51 \mathrm{M}$ & SM Grade 1 parietal AVM; ruptured & Elective & 3 \\
\hline 32 & $55 \mathrm{~F}$ & A-comm aneurysm & Emergency & 2 \\
\hline 33 & $44 \mathrm{M}$ & Insular cavernoma & Elective & 1 \\
\hline 34 & $77 \mathrm{~F}$ & A-comm aneurysm & Elective & 2 \\
\hline 35 & $41 \mathrm{M}$ & SM Grade 3 torcula AVM; unruptured & Elective & 3 \\
\hline 36 & $64 \mathrm{~F}$ & Complex MCA aneurysm - awake & Elective & 3 \\
\hline 37 & $46 \mathrm{~F}$ & Temporo-parietal GBM - awake & Elective & 1 \\
\hline 38 & $38 \mathrm{M}$ & A-comm aneurysm & Emergency & 2 \\
\hline 39 & $55 \mathrm{~F}$ & Proximal M1 aneurysm & Elective & 2 \\
\hline 40 & $54 \mathrm{M}$ & SM Grade 2 Parieto-occipital AVM & Elective & 3 \\
\hline 41 & $59 \mathrm{~F}$ & MCA aneurysm & Elective & 2 \\
\hline 42 & $46 \mathrm{~F}$ & MCA aneurysm & Elective & 2 \\
\hline 43 & $50 \mathrm{M}$ & MCA aneurysm & Elective & 2 \\
\hline 44 & $65 \mathrm{~F}$ & OA and MCA aneurysms & Elective & 2 \\
\hline 45 & $23 \mathrm{~F}$ & Falcine meningioma & Elective & 3 \\
\hline 46 & $47 \mathrm{~F}$ & MCA aneurysm & Elective & 2 \\
\hline
\end{tabular}

* "Benefit": $1=$ Not useful $(\mathrm{n}=11 ; 24 \%) 2=$ Useful but did not influence surgical management $(\mathrm{n}=22 ; 48 \%) 3=$ Useful and influenced surgical management $(n=12 ; 26 \%) 4=$ False reassurance $(n=1 ; 2 \%)$. 
to the fluorescence image during fluorescence mode. For the benefit of the anaesthetists, theatre nurses, junior medical officers and medical students, the fluorescence image was transmitted to a mobile screen mounted on the Leica $\mathrm{OH} 4$ microscope or an external viewing device connected to the microscope via an S-video cable. Touch screen functionality on the microscope LCD screen allowed recorded data loops to be played back at any point of time for reassessment of the intra-operative findings.

In contrast to intra-operative DSA, there was no need for additional bulky imaging apparatus. No additional staff such as radiographers or interventional radiologists were required during the operation. The technique required a simple intravenous injection of ICG and the cerebral vasculature was visualised with the same microscope switched to its fluorescence mode. The arterial phase was visualised 25-35 seconds after ICG injection with the venous phase over the subsequent 30 seconds.

\section{Resolution of images}

The neurosurgeon found that the image resolution was comparable, if not superior, to DSA. The quality and resolution of the images was excellent, with arterioles of approximately 100 micrometres visible (Fig. 1). Importantly, it allowed the surgeon to visualise and thus preserve the vital perforating arteries during the operation. The tissue penetrance of the fluorescence was, as expected, extremely limited compared to X-ray. Unlike DSA, ICGFV could only visualise a small area of exposed cerebral vasculature. The area and depth of the illuminated operating field was limited by the amount of surgical exposure and magnification. Structures such as the muscles or brain parenchyma obstructing the line-ofsight precluded visualisation of the underlying fluorescing vascular structures. Blood or substantial fluid in the operating field would also obscure the intended visualization. As a result, the success of the fluorescence was greatly enhanced by careful attention to haemostasis prior to imaging.

\section{Clinical benefit}

The use of intra-operative ICGFV benefited a total of 34 of 46 cases (74\%; Table II). The fluorescence image allowed delineation of the cerebrovascular anatomy before and after the clipping of aneurysms, the progressive resection of AVM nidi, and the disconnection of DAVF. ICGFV demonstrated successful aneurysm clipping (absence of fluores-
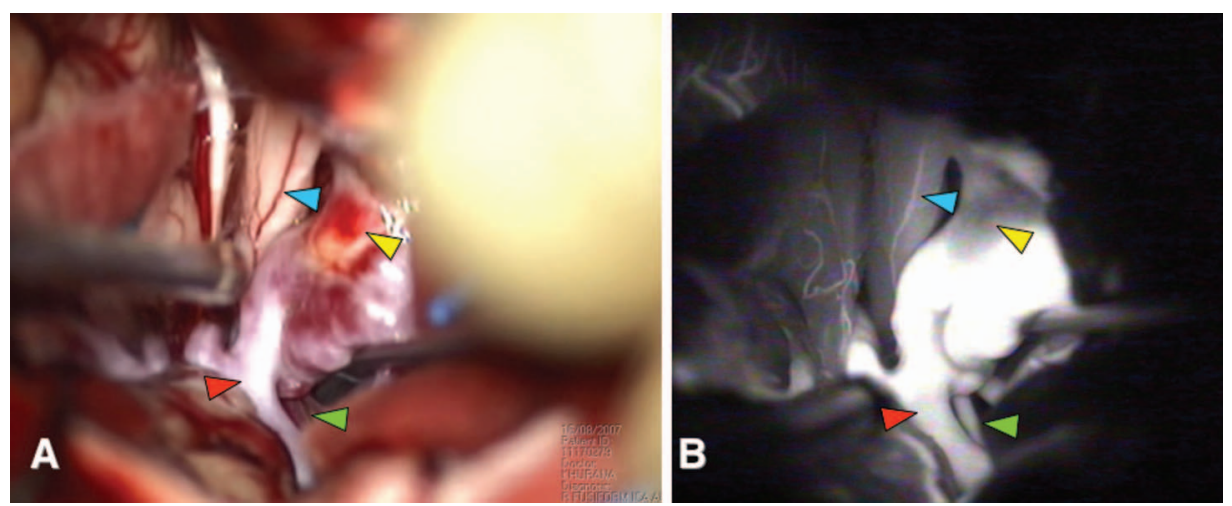

FIG. 1. View through the eyepieces shortly after intravenous ICG administration. In fluorescence mode, immediately prior to clip reconstruction of this fusiform proximal internal carotid artery aneurysm, one eye piece shows the operative anatomy (A) while simultaneously the other eye piece shows the anatomy in fluorescence mode (B). Note the following: Atheroma in the aneurysm (yellow arrow head); carotid artery bifurcation (red arrow head); a previously poorly visible anterior choroidal artery (green arrow head) becomes clearly visible in fluorescence mode; even small nutrient arterioles on the optic nerve (blue arrow head) can be seen, demonstrating the very sharp resolution of ICG at the arteriolar (and perforator) level.

TABLE II. Clinical benefit and utility of ICGFV

\begin{tabular}{|c|c|c|c|c|c|}
\hline Craniotomy for: & Not useful & Useful but did not influence surgery & Useful and influenced surgery & False Reassurance & Total \\
\hline Aneurysm & 0 & 21 & 6 & 0 & 27 \\
\hline AVM & 1 & 0 & 3 & 1 & 5 \\
\hline DAVF & 0 & 0 & 1 & 0 & 1 \\
\hline EC-IC bypass & 0 & 1 & 1 & 0 & 2 \\
\hline Cavernoma & 3 & 0 & 0 & 0 & 3 \\
\hline Meningioma & 4 & 0 & 1 & 0 & 5 \\
\hline Glioma & 3 & 0 & 0 & 0 & 3 \\
\hline Total & $11(24 \%)$ & $22(48 \%)$ & $12(26 \%)$ & $1(2 \%)$ & 46 \\
\hline
\end{tabular}

AVM, arteriovenous malformation; DAVF, dural arteriovenous fistula; EC-IC, extracranial-intracranial. 
cence in the aneurysm lumen) without inadvertent compromise to the distal vessel flow (preserved fluorescence in distal vessels including neighbouring perforators) (Fig. 2). ICGFV provided reassurance that the shutdown of an AVM was successful (no fluorescence within the nidus) prior to occluding the draining veins (Fig. 3).

ICGFV was found to be particularly useful in emergency surgeries involving ruptured or complex aneurysms where the aneurysm anatomy and relevant pre- and post- vascular anatomy was difficult to visualise directly. Surgery for most of the simple saccular unruptured aneurysms undergoing elective clipping did not substantially benefit from ICGFV, as the pertinent vascular anatomy was evident by direct visualisation. It was, however, better demonstrated under fluorescence. In the two cases of EC-IC bypass in our series, ICGFV confirmed the patency of the radial artery conduit with robust antegrade flow at the conclusion of the anastomoses (Fig. 4).

In 12 of the 46 cases ( $26 \%$, Table II), ICGFV led to crucial intra-operative surgical changes. Among the 27 aneurysm surgeries, inadvertent parent vessel occlusion was detected in 1 case ( $4 \%$; craniotomy 2 in Table I), inadvertent adjacent main arterial branch occlusion was detected in 1 case $(4 \%$; craniotomy 3 in Table I) and incomplete aneurysm neck occlusion with residual filling of the fundus was detected in 2 cases ( $7 \%$; craniotomies 10 and 23 in Table I). In a further 2 cases (7\%; craniotomies 26 and 36 in Table I), ICGFV played a key role in facilitating optimal clip reconstruction of complex aneurysms such that distal flow was assured and unclipable residual necks were reinforced with Teflon wrapping (Fig. 2).

In craniotomy 17 (Table I, Fig. 5), the diagnosis of a cortical DAVF was made intra-operatively using ICGFV. Pre-operative DSA was not definitive. The surgeon identified abnormal arterial-phase fluorescence in a cortical vein in the centre of the hematoma. The cortical DAVF was then disconnected successfully. ICGFV was used to confirm the successful disconnection of this anomaly. Postoperative DSA demonstrated no residual DAVF. In AVM excisions (craniotomies 31, 35 and 40 in Table I; see Fig. 3), progressive shutdown of the nidus was clearly demonstrated by serial ICGFV. In one patient with a giant meningioma involving the wall of the superior sagittal sinus (craniotomy 45 in Table I), delayed intraoperative cerebral oedema was noted. ICGFV was used to visualise flow in the superior sagittal sinus and adjoining veins. Sluggish venous flow in the tributary cortical veins was observed, suggesting sinus obstruction, which was corrected by releasing the dural flap retraction over the sinus. The oedema settled.

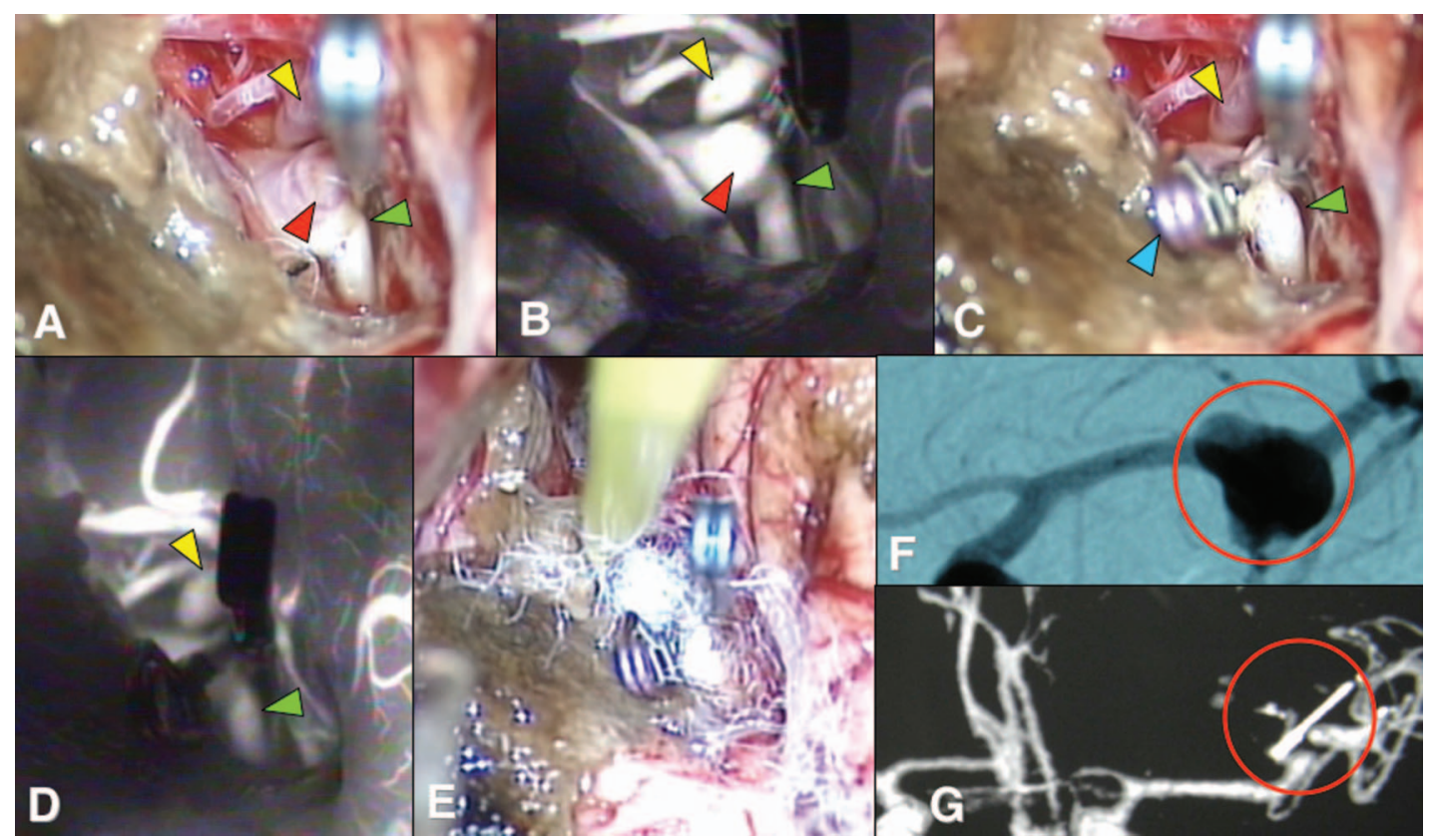

FIG. 2. An example of the usefulness of ICGFV during clip reconstruction of a fusiform, atherosclerotic 10 mm middle cerebral artery aneurysm (craniotomy 36 in Table I). (A) The first clip has been placed on part of the aneurysm sac (red arrow head). The temporal (yellow arrow head) and frontal (green arrow head) M2 branches are seen. The frontal branch is very atherosclerotic, and the surgeon is concerned that flow in the branch has been compromised by the first clip (kink at frontal M2 origin; tip of green arrow head). (B) ICG confirms good distal flow in both M2 branches (yellow and green arrow heads), and residual filling in the unclipped portion of the aneurysm sac, as expected (red arrow head). The clip did not need to be readjusted. (C) A second clip (blue arrow head) is placed on the residual sac. Flow in especially the atherosclerotic frontal M2 branch (green arrow head) may be compromised by placement of this second clip. (D) However, ICG shows excellent filling in both M2 branches at this time. (E) Teflon microfibers are placed around the collapsed sac to assist in its long-term obliteration. (F) Preoperative catheter angiogram showing the fusiform middle cerebral artery aneurysm prior to clipping (red circle). (G) Postoperative CTA showing no residual filling of the aneurysm and patency of the M2 branches. The patient experienced no deficit from surgery. 

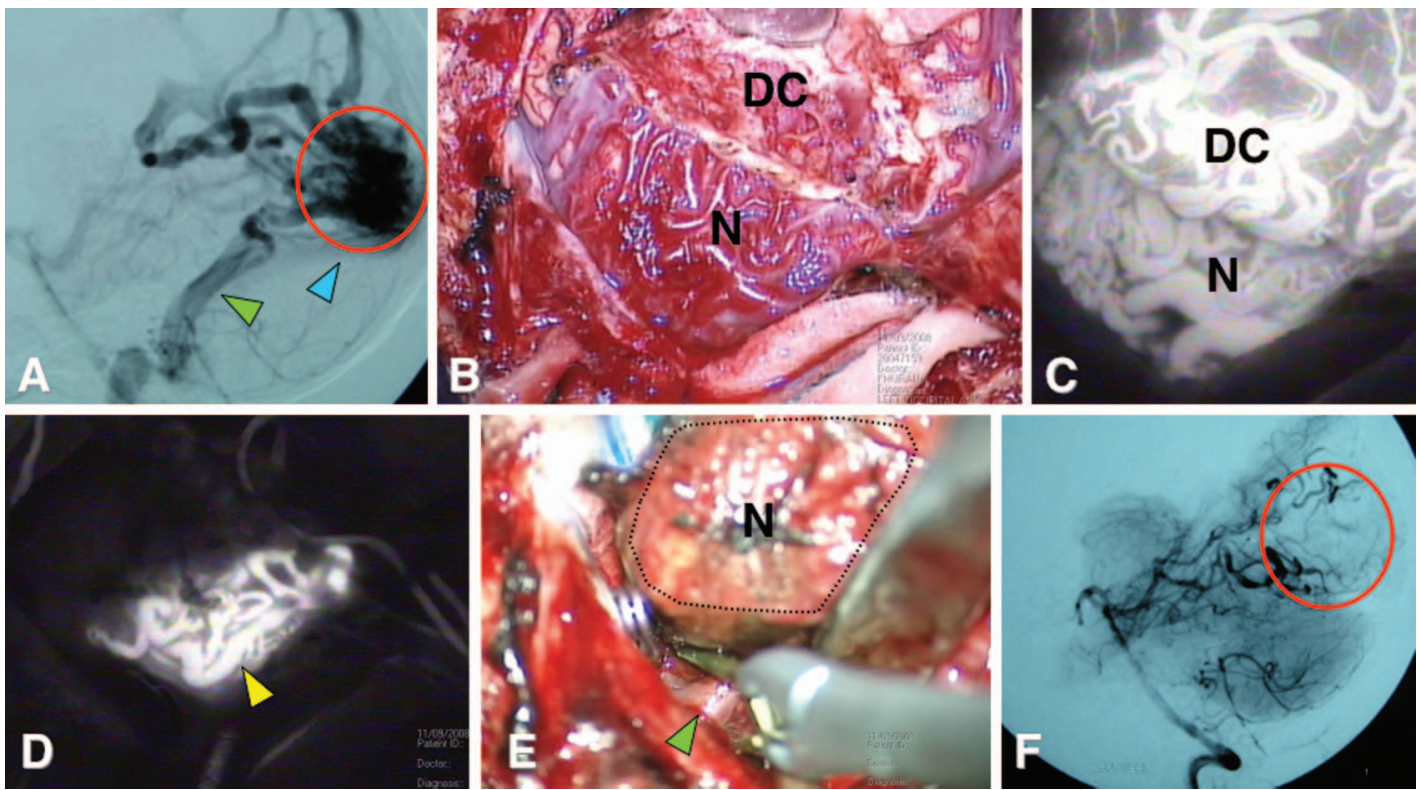

FIG. 3. An example of the usefulness of ICGFV during AVM excision. (A) The patient (craniotomy 35 in Table I) presented with a symptomatic torcula region occipital AVM (Spetzler-Martin grade 3). The nidus is shown in the red circle of this preoperative catheter angiogram (vertebral artery injection, lateral projection). The transverse (blue arrow head) and sigmoid (green arrow head) venous sinuses are also shown. (B) Intraoperative view of the nidus (N) and dilated cortical vessels (DC) adjacent to the nidus. (C) Fluorescence view of the same. (D) As the arterial supply to the nidus is gradually shut down surgically, ICGFV shows whatever blood flow remains (yellow arrow head) in the progressively obliterating nidus. (E) The nidus $(\mathrm{N})$ has been circumferentially dissected now, and a final clip is placed on a major AVM vein (green arrow head) draining into the torcula. (F) Postoperative catheter angiogram (vertebral artery injection, lateral projection) shows complete excision of the nidus (red circle), but some surrounding dilated cortical veins are noted as expected following resection of this high-flow AVM. The patient has made an excellent postoperative recovery.

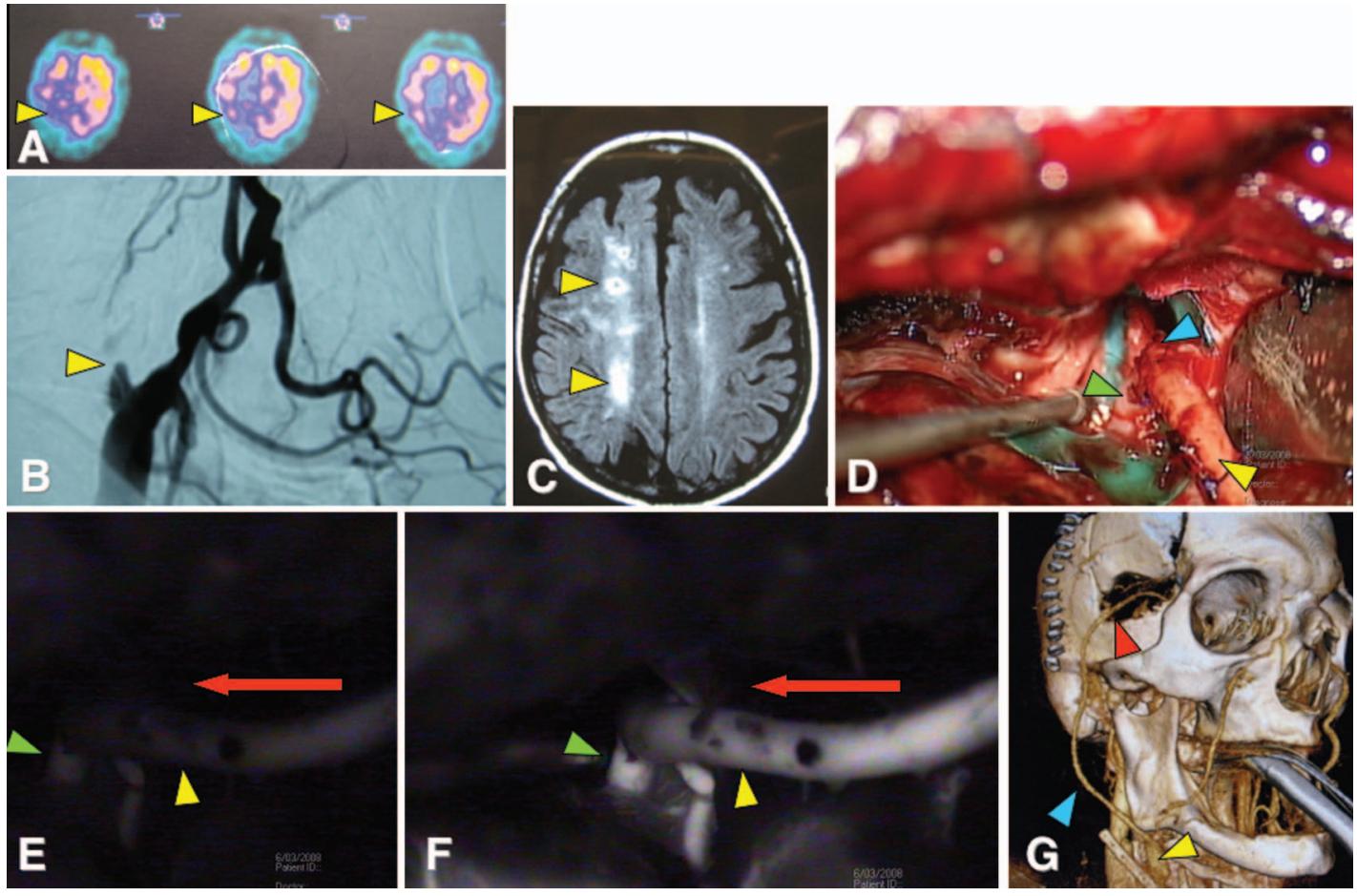

FIG. 4. An example of the usefulness of ICGFV during EC-IC bypass for symptomatic right internal carotid artery (ICA) occlusion (craniotomy 24 in Table I). (A) Radionucleide scan during acetazolamide challenge shows right hemispheric hypoperfusion (yellow arrow head). (B) Catheter angiogram shows tapered occlusion at the origin of the right ICA (yellow arrow head). (C) MRI fluid-attenuated inversion recovery (FLAIR) sequence shows evidence for right hemisphere-predominant cerebral ischaemia (yellow arrow heads). (D) Intraoperative image shows a radial artery bypass graft (yellow arrow head) surgically anastomosed (blue arrow head) with the right middle cerebral artery M2 frontal branch (green arrow head). (E) At the conclusion of the surgery, fluorescence imaging confirms patent antegrade flow along the radial artery graft (yellow arrow head) towards the M2 branch (green arrow head) as the graft's fluorescence intensifies in the antegrade direction of the red arrow. (F) In time, the fluorescence intensifies in the same antegrade direction. (G) Early postoperative 3D CTA (patient still intubated for strict cerebral perfusion control) shows the subcutaneous radial artery graft (blue arrow head) entering into the small craniotomy (red arrow head). The radial artery has been sutured proximally to the external carotid artery (yellow arrow head). The patient remains asymptomatic and neurologically intact now more than a year after the operation. 

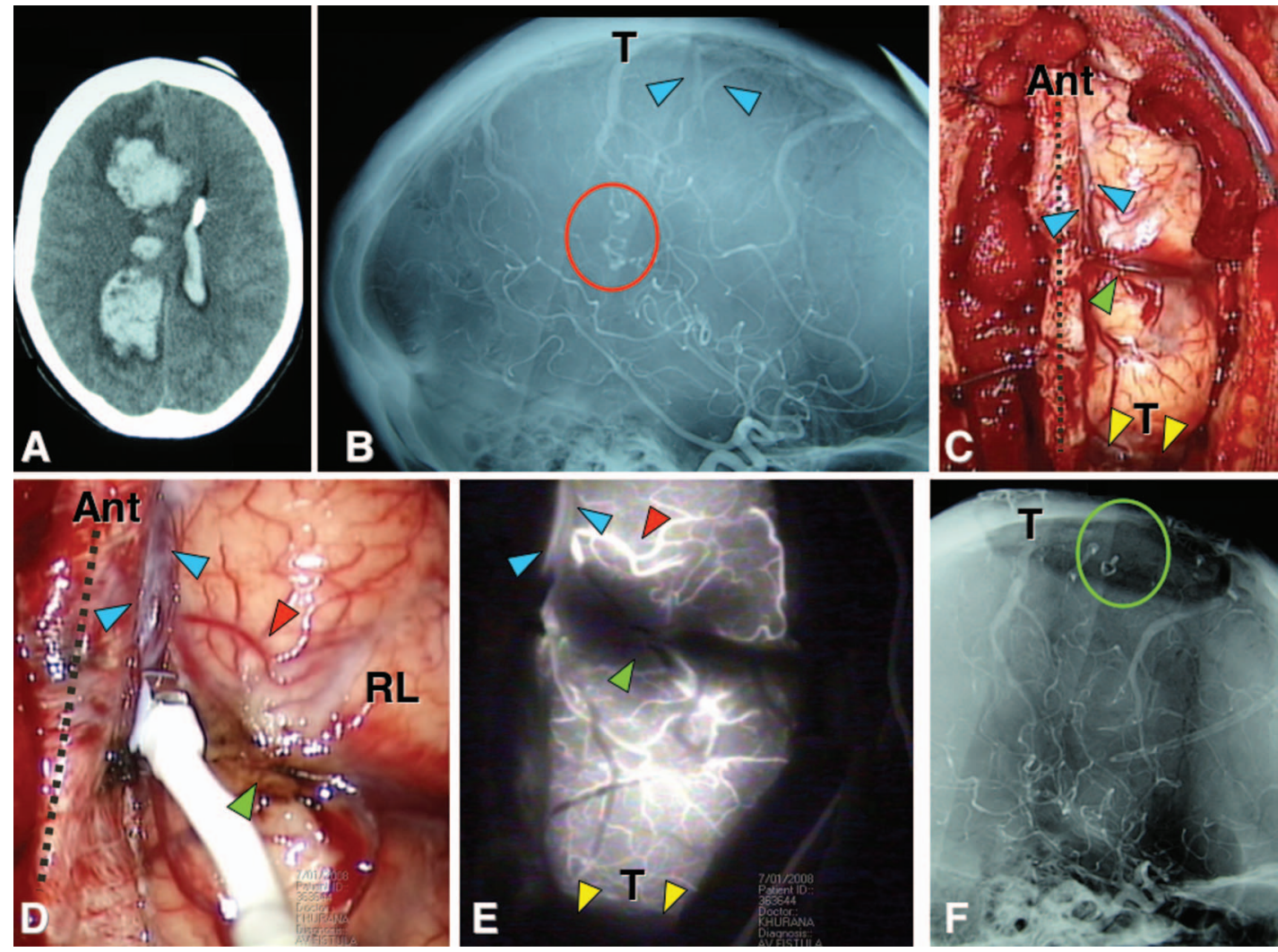

FIG. 5. An example of the usefulness of ICGFV during DAVF disconnection. (A) The patient (craniotomy 17 in Table I) was moribund preoperatively from a large intracranial haemorrhage. (B) Catheter angiography revealed dysplastic vessels (red circle) associated with a complex of early filling veins (blue arrow heads) anterior to the vein of Trolard (T), all draining into the superior sagittal sinus. (C) Intraoperative image following right parasagittal craniotomy showing the Trolard vein ( $T$; yellow arrow heads), and what are presumed to be the early filling veins (blue and green arrow heads). (D) Intraoperative ultrasonographic flowmetry ${ }^{30}$ reveals high blood volume flow rates through these veins (blue arrow heads) indicating arterialization. An arteriole is denoted by the red arrow head. One of the veins (green arrow head) has been obliterated by cautery. (E) As shown in this arteriolar phase (red arrow head) of the fluorescence imaging intraoperatively, the suspicious veins (blue arrow heads) fluoresce early compared with the late-filling Trolard vein ( $T$; yellow arrow heads). The early filling veins are cauterised then clipped. (F) Postoperative catheter angiography showing titanium clip and no further filling of the early draining veins (green circle). The dysplastic vessels seen in B are no longer evident. Following rehabilitation, the patient made an excellent neurological recovery. Dotted line $=$ superior sagittal sinus dura; $\mathrm{Ant}=$ anterior, $\mathrm{RL}=$ right lateral.

\section{False reassurance}

In all but one patient in our series, the ICGVF findings intraoperatively were compatible with those based on postoperative angiography. The exceptional patient (craniotomies 12 and 13 in Table I) had presented with a large intraparenchymal haemorrhage with intraventricular extension secondary to a Spetzler-Martin grade 3 left insular AVM with associated intraventricular venous aneurysm. A craniotomy was performed to shut down the AVM. With the use of ICGFV, the arterial feeding vessels were thought to be shut down as no residual fluorescence was observed in the nidus. Unfortunately, post-operative DSA showed a residual feeding artery and the remaining partial nidus adjacent to the original excision. A second operation was performed to complete the shutdown of the AVM. In reviewing the reasons why ICGFV failed to demonstrate the persisting AVM, it became evident that the residual arterial feeder and nidus lay under brain parenchyma at one margin of the previous operative field. It is likely that this residual nidus would have been detected by intraoperative DSA.

\section{Benefit in non-cerebrovascular neurosurgical cases}

With the exception of one case (patient 45 in Table I; see above), there was no benefit using ICGFV in the remaining non-cerebrovascular cases $(n=7)$ or for cavernous malformations $(n=3)$. The surgeon had selectively chosen patients with large intracranial tumours that lay adjacent to critical vascular structures such as the superior sagittal sinus, basilar artery or the anterior cerebral artery. The surgeon intended to determine if the use of ICGFV could enhance the safety of the operation by serial visualisation of the vital vascular structures, thus preventing inadvertent surgical retraction or dissection. The surgeon performed ICGFV in 11 cases (5 cases of meningioma, 3 cases of astrocytoma and 3 cases of cavernous malformation) and found that the use of ICGFV in all but one case provided no benefit to the standard operating procedure. That is, direct visualisation using the conventional surgical microscope was generally adequate for such purposes. Interestingly, there was no fluorescence seen in small developmental venous anomalies associated with all three cavernous malformations, 
indicating no active flow in these small venous structures (Fig. 6)

\section{Discussion}

In aneurysm surgery, shortcomings such as residual aneurysm neck (3.1-47.0\%), distal artery stenosis $(0.3-44.0 \%)$ and post-surgical infarction (0.8$33.0 \%$ ) have been well documented. ${ }^{1,9-18}$ Johnston et $a l .{ }^{19}$ reported in their recent CARAT study that there was a strong correlation between aneurysm rerupture and the degree of post-clipping occlusion. Successful aneurysmal neck clipping results in a good long-term angiographic outcome. ${ }^{20}$ In order to avoid the aforementioned complications and to enhance the success of the aneurysm surgery, many authors have suggested the use of routine intra-operative angiography in the majority of, if not all, aneurysm cases. ${ }^{9,10,12,17,21}$

Unfortunately, intra-operative cerebral angiography is not readily available in many centres, including our own. Intra-operative angiography requires additional equipment set-up, a radiographer, an interventional radiologist, more theatre space, and other logistic support. It is an invasive procedure asso- ciated with unlikely but important complications such as focal neurological deficit secondary to embolic CVA and lower limb ischaemia secondary to femoral artery thrombosis $(0.4-2.6 \%) .^{22}$ To improve cost-effectiveness and benefit-risk ratio, it has been suggested that intra-operative DSA be used selectively. Selection criteria include cases with intraoperative ruptured aneurysm, large broad-based complex aneurysms, posteriorly located aneurysms or proximal ICA aneurysms. ${ }^{11,13-17,22-25}$

Intra-operative DSA also requires a substantial time to catheterise the vessel of interest. It also exposes any unshielded staff to X-ray radiation. An experienced team would require at least 20-30 minutes to perform intra-operative angiography. The time delay is crucial in the setting of cerebral perfusion and oxygenation. Previous authors have indicated a $33 \%$ rate of stroke after clip readjustment to restore flow into occluded vessels seen on intraoperative DSA. ${ }^{4}$ Thus, a fast, accurate and readily available imaging modality is highly desirable in demonstrating parent arterial patency, adequate aneurysm obliteration and distal artery flow.

Intra-operative ICGFV fulfils these criteria. The authors found that ICGFV is quick, easy and
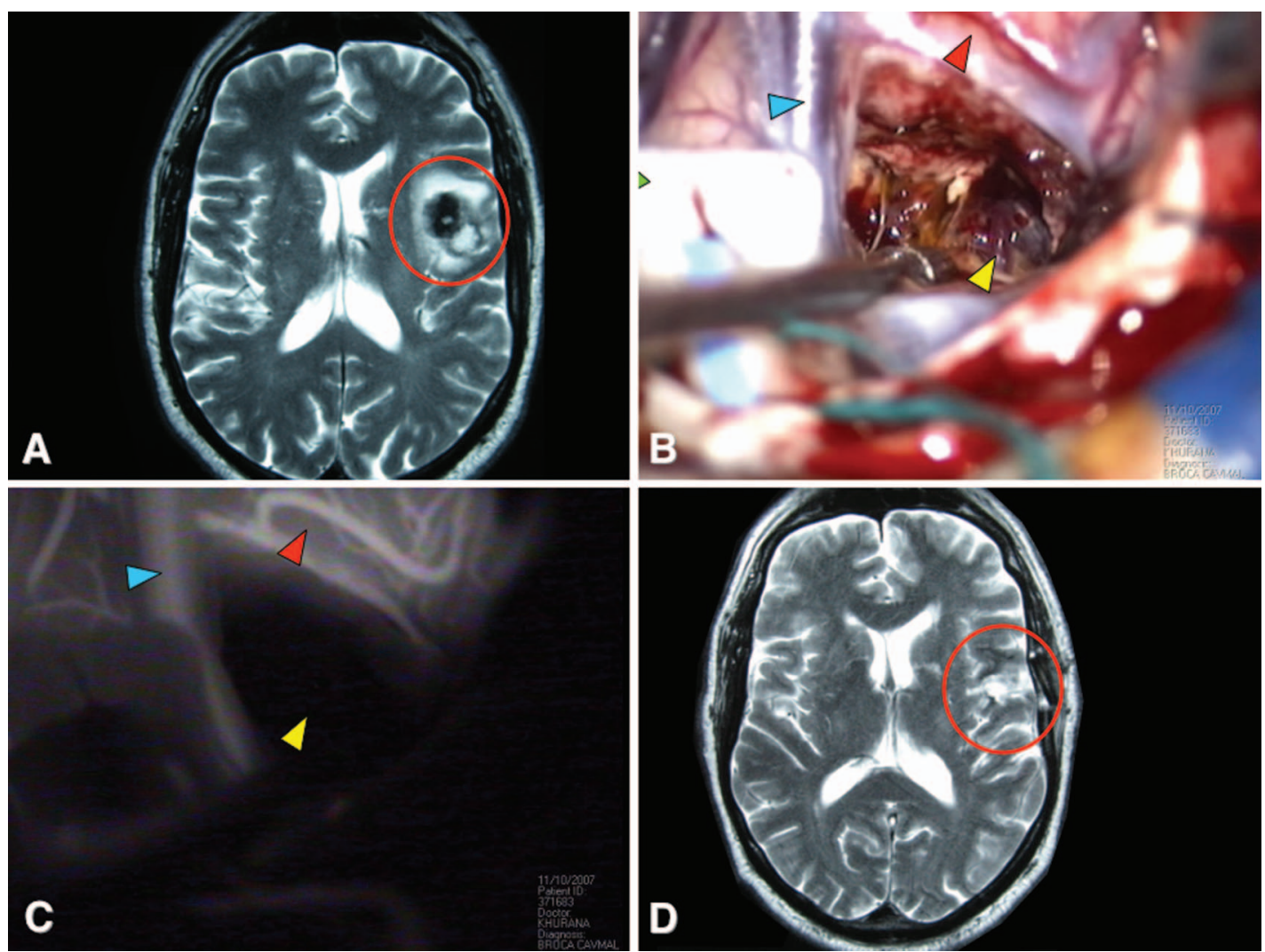

FIG. 6. Demonstration of the lack of usefulness of ICGFV during cavernoma surgery (craniotomy 9 in Table I). A. This expanding cavernoma in the patient's Broca area had presented with recurrent spells of expressive aphasia. T2-weighted axial MR image shown. The patient underwent awake craniotomy. B. Intraoperative image showing hemosiderin staining and a venous anomaly (yellow arrow head) associated with the cavernous sacs. Surrounding pial arteries (red arrow head) and veins (blue arrow head) are noted. C. In fluorescence mode, the same pial vessels are seen (red and blue arrow heads), but no vessels are fluorescing in the bed of the cavernoma at this time (yellow arrow head), i.e., prior to its resection and despite identification of a venous anomaly in the same field of view. D. Postoperative T2weighted axial MR image showing complete resection of the cavernoma. The patient remains asymptomatic and neurologically intact over 1 -year postoperatively. 
intuitive, consistent with the experience of others. ${ }^{2-4}$ Its real-time high-resolution images are adequate to visualise and preserve the small but important perforating arteries. ${ }^{1,5}$ Besides aneurysm clipping, various cerebrovascular cases such as EC-IC bypass ${ }^{7}$, DAVF disconnection and AVM excision ${ }^{6}$ could benefit from the use of intra-operative ICGFV. In awake craniotomy cases, five of which were included in our study, there was no reported discomfort during the ICG injection. In none of our 46 cases was there any side-effect from ICG administration as per our protocol.

Raabe et $a .^{4}$ demonstrated that although intraoperative DSA provides the most accurate and therefore the gold standard for intra-operative cerebrovascular imaging, intra-operative ICGFV is both useful as an independent form of angiography or as an adjunct to intra- or post-operative DSA. Its routine use in aneurysm clipping is highly recommended, especially in centres where intra-operative angiography is not available. We found that our results with intra-operative ICGFV were highly reliable when the same patients were imaged using DSA and/or CTA post-operatively. As described earlier, the only postoperative angiographic finding incompatible with the intraoperative ICGVF finding was that of a small residual nidus in an AVM patient. It is in complex AVM surgery where intraoperative DSA may still hold an advantage over intraoperative ICGFV.

\section{Limitations of ICG}

The authors recognise that ICGFV is qualitative rather than quantitative. It is conceivable that despite observing what appears to be good distal vessel fluorescence in the relevant vessels, ICGFV might not demonstrate diminished volume flow in these vessels. We and others have demonstrated the safety and usefulness of intracranial transit time flowmetry (ITTF) and microvascular Doppler ultrasonography, and have reported that both techniques are cost-effective in enhancing safety and reducing the risk of post-operative stroke in cerebrovascular surgery. ${ }^{26-32}$ Our preference for ultrasound-based flow modalities is ITTF (quantitative) ${ }^{30}$ rather than non-quantitative Doppler. Despite the limitation that ICGFV is non-quantitative in terms of blood flow measurement, we found that it still reliably predicted post-operative clinical and radiological outcome.

Compared to DSA, the area and depth of ICGFV visualization is limited, especially in circumstances such as excision of a large AVM. This limitation is due to the amount of surgical exposure or any physical obscuration of the 'line-of-sight' between the fluorescing vasculature and the microscopemounted NIR camera. Within these important limitations, Takagi et al. ${ }^{6}$ demonstrated that the use of ICGFV is a safe and easy method for assessing the completeness of AVM excision. In our limited experience, ICGFV should be used in more technically difficult AVM and DAVF cases in conjunction with intra-operative or post-operative DSA in the future.

\section{Conclusion}

ICGFV is an intuitive method that provides neurosurgeons with high-quality, reliable, real-time information regarding cerebrovascular anatomy, which can assist in improving intra-operative surgical management and stroke prevention. It has technical limitations that need to be understood. We cannot overemphasise the potential for enhancement of patient safety in those undergoing high-risk neurosurgical procedures using this emerging technology. We believe that this work demonstrates the utility of this technology in a wide array of intracranial procedures such as aneurysm, AVM, DAVF and EC-IC bypass cases and during awake craniotomy. The technology was not found to be useful in cavernoma surgery.

\section{Acknowledgements and Declaration of Interests}

The contributions by the authors to this paper were as follows: VGK and KS wrote the paper. DD developed this hospital's protocol for intraoperative ICG administration and edited this paper. VGK and KS added the relevant data prospectively to a database. The authors thank Professor Paul Gatenby, Director of Research at The Canberra Hospital for his support. The authors also gratefully acknowledge the assistance of The Canberra Hospital Pharmacy staff and Ms Vicki Gardiner, Ms Kerri Reeves, Ms Rhonda Hosking, Ms Tania Lawrence, and $\mathrm{Mr}$ Frank Byrne. There were no sources of funding from grant bodies or Industry in order to undertake this research. The authors duly acknowledge the expert technical assistance of the staff of Leica Microsystems and Medtronic Australasia in optimisation of the equipment and software.

\section{References}

1 de Oliveira JG, Beck J, Seifert Teixeira MJ, Raabe A. Assessment of flow in perforating arteries during intracranial aneurysm surgery using intraoperative near-infrared indocyanine green videoangiography. Neurosurgery 2007;61(3 Suppl); 63-72.

2 Raabe A, Beck J, Gerlach R, Zimmermann M, Seifert V. Nearinfrared indocyanine green video angiography: a new method for intraoperative assessment of vascular flow. Neurosurgery 2003;52:132-9.

3 Raabe A, Beck J, Seifert V. Technique and image quality of intraoperative indocyanine green angiography during aneurysm surgery using surgical microscope integrated near-infrared video technology. Zentralbl Neurochir 2005;66:1-6.

4 Raabe A, Nakaji P, Beck J, Kim LJ, Hsu FP, Kamerman JD, Seifert V, Spetzler RF. Prospective evaluation of surgical microscope-integrated intraoperative near-infrared indocyanine green videoangiography during aneurysm surgery. $\mathcal{F}$ Neurosurg 2005;103:982-9. 
5 Suzuki K, Kodama N, Sasaki T, Matsumoto M, Ichikawa T, Munakata R, Muramatsu H, Kasuya H. Confirmation of blood flow in perforating arteries using fluorescein cerebral angiography during aneurysm surgery. $\mathcal{F}$ Neurosurg 2007;107:68-73.

6 Takagi Y, Kikuta KI, Nozaki K, Sawamura K, Hashimoto N. Detection of a residual nidus by surgical microscope-integrated intraoperative near-infrared indocyanine green videoangiography in a child with a cerebral arteriovenous malformation. $\mathcal{F}$ Neurosurg 2007;107:416-8.

7 Woitzik J, Horn P, Vajkoczy P, Schmiedek P. Intraoperative control of extracranial-intracranial bypass patency by nearinfrared indocyanine green videoangiography. $f$ Neurosurg 2005;102:692-8.

8 Wrobel CJ, Meltzer H, Lamond R, Alksne JF. Intraoperative assessment of aneurysm clip placement by intravenous fluorescein angiography. Neurosurgery 1994;35:970-3.

9 Carvi y Nievas MN. Assessment of the clipping efficacy of intracranial aneurysms: analysis of the employed methodology in relation to case difficulty. Neurol Res 2007;29:506-16.

10 Chiang VL, Gailloud P, Murphy KJ, Rigamonti D, Tamargo RJ. Routine intraoperative angiography during aneurysm surgery. F Neurosurg 2002;96:988-92.

11 Katz JM, Gologorsky Y, Tsiouris AJ, Wells-Roth D, Mascitelli J, Gobin YP, Stieg PE, Riina HA. Is routine intraoperative angiography in the surgical treatment of cerebral aneurysms justified? A consecutive series of 147 aneurysms. Neurosurgery 2006;58:719-27.

12 Meyer B, Urbach H, Schaller C, Baslam M, Nordblom J, Schramm J. Immediate postoperative angiography after aneurysm clipping - implications for quality control and guidance of further management. Zentralbl Neurochir 2004;65:49-56.

13 Nanda A, Willis BK, Vannemreddy PS. Selective intraoperative angiography in intracranial aneurysm surgery: intraoperative factors associated with aneurysmal remnants and vessel occlusions. Surg Neurol 2002;58:309-14.

14 Payner TD, Horner TG, Leipzig TJ, Scott JA, Gilmor RL, DeNardo AJ. Role of intraoperative angiography in the surgical treatment of cerebral aneurysms. F Neurosurg 1998;88:441-8.

15 Popadic A, Witzmann A, Amann T, Doringer W, Fleisch M, Häfel C, Hergan $K$, Längle $M$. The value of intraoperative angiography in surgery of intracranial aneurysms: a prospective study in 126 patients. Neuroradiology 2001;43:466-71.

16 Rauzzino MJ, Quinn CM, Fisher WS, 3rd. Angiography after aneurysm surgery: indications for "selective" angiography. Surg Neurol 1998;49:32-40.

17 Tang G, Cawley CM, Dion JE, Barrow DL. Intraoperative angiography during aneurysm surgery: a prospective evaluation of efficacy. F Neurosurg 2002;96:993-9.

18 Thornton J, Bashir Q, Aletich VA, Debrun GM, Ausman JI, Charbel FT. What percentage of surgically clipped intracranial aneurysms have residual necks? Neurosurgery 2000;46:1294-8.

19 Johnston SC, Dowd CF, Higashida RT, Lawton MT, Duckwiler GR, Gress DR; CARAT Investigators. Predictors of rehemorrhage after treatment of ruptured intracranial aneurysms: The Cerebral Aneurysm Rerupture After Treatment (CARAT) study. Stroke 2008;39:120-5.

20 Akyüz M, Tuncer R, Yilmaz S, Sindel T. Angiographic followup after surgical treatment of intracranial aneurysms. Acta Neurochir (Wien) 2004;146:245-50.

21 Klopfenstein JD, Spetzler RF, Kim LJ, Feiz-Erfan I, Han PP, Zabramski JM, Porter RW, Albuquerque FC, McDougall CG, Fiorella DJ. Comparison of routine and selective use of intraoperative angiography during aneurysm surgery: a prospective assessment. F Neurosurg 2004;100:230-5.

22 Martin NA, Bentson J, Viñuela F, Hieshima G, Reicher M, Black K, Dion J, Becker D. Intraoperative digital subtraction angiography and the surgical treatment of intracranial aneurysms and vascular malformations. F Neurosurg 1990;73:52633.

23 Alexander TD, Macdonald RL, Weir B, Kowalczuk A. Intraoperative angiography in cerebral aneurysm surgery: a prospective study of 100 craniotomies. Neurosurgery 1996; 39:10-17.

24 Derdeyn CP, Moran CJ, Cross DT, 3rd, Sherburn EW, Dacey RG Jr. Intracranial aneurysm: anatomic factors that predict the usefulness of intraoperative angiography. Radiology 1997;205: 335-9.

25 Yanaka K, Asakawa H, Noguchi S, Matsumaru Y, Hyodo A, Anno I, Meguro $\mathrm{K}$, Nose $\mathrm{T}$. Intraoperative angiography evaluation of the microsurgical clipping of unruptured cerebral aneurysms. Neurol Med Chir (Tokyo) 2002;42:193-200.

26 Akdemir H, Oktem IS, Tucer B, Menkü A, Basaslan K, Günaldi O. Intraoperative microvascular Doppler sonography in aneurysm surgery. Minim Invasive Neurosurg 2006;49:312-6.

27 Amin-Hanjani S, Meglio G, Gatto R, Bauer A, Charbel FT. The utility of intraoperative blood flow measurement during aneurysm surgery using an ultrasonic perivascular flow probe. Neurosurgery 2006;58(4 Suppl 2):ONS-305-12.

28 Bailes JE, Tantuwaya LS, Fukushima T, Schurman GW, Davis D. Intraoperative microvascular Doppler sonography in aneurysm surgery. Neurosurgery 1997;40:965-70.

29 Fagundes-Pereyra WJ, Hoffman WE, Misra M, Charbel FT. Clip readjustment in aneurysm surgery after flow evaluation using the ultrasonic perivascular probe: case report. Arq Neuropsiquiatr 2005;63(2A):339-44.

30 Kirk HJ, Rao PJ, Seow K, Fuller J, Chandran N, Khurana VG. Intraoperative transit time flowmetry reduces the risk of ischaemic neurological deficits in Neurosurgery. Br $\mathcal{F}$ Neurosurg 2009;23:40-7.

31 Marchese E, Albanese A, Denaro L, Vignati A, Fernandez E, Maira G. Intraoperative microvascular Doppler in intracranial aneurysm surgery. Surg Neurol 2005;63:336-42.

32 Stendel R, Pietilä T, Al Hassan AA, Schilling A, Brock M. Intraoperative microvascular Doppler ultrasonography in cerebral aneurysm surgery. F Neurol Neurosurg Psych 2000;68: 29-35. 